\title{
Representation of a Solution of the Cauchy Problem for an Oscillating System with Multiple Delays and Pairwise Permutable Matrices
}

\author{
Josef Diblík, ${ }^{1}$ Michal Fečkan, ${ }^{2,3}$ and Michal Pospíšil ${ }^{4}$ \\ ${ }^{1}$ Department of Mathematics, Faculty of Electrical Engineering and Communication, Brno University of Technology, \\ Technická 3058/10, 61600 Brno, Czech Republic \\ ${ }^{2}$ Department of Mathematical Analysis and Numerical Mathematics, Comenius University, Mlynská dolina, \\ 84248 Bratislava, Slovakia \\ ${ }^{3}$ Mathematical Institute of Slovak Academy of Sciences, Štefánikova 49, 81473 Bratislava, Slovakia \\ ${ }^{4}$ Centre for Research and Utilization of Renewable Energy, Faculty of Electrical Engineering and Communication, \\ Brno University of Technology, Technická 3058/10, 61600 Brno, Czech Republic
}

Correspondence should be addressed to Michal Pospísil; pospisilm@feec.vutbr.cz

Received 13 January 2013; Accepted 19 April 2013

Academic Editor: Jaan Janno

Copyright (c) 2013 Josef Diblík et al. This is an open access article distributed under the Creative Commons Attribution License, which permits unrestricted use, distribution, and reproduction in any medium, provided the original work is properly cited.

Nonhomogeneous system of linear differential equations of second order with multiple different delays and pairwise permutable matrices defining the linear parts is considered. Solution of corresponding initial value problem is represented using matrix polynomials.

\section{Introduction}

Motivated by delayed exponential representing a solution of a system of differential or difference equations with one or multiple fixed or variable delays [1-6], which has many applications in theory of controllability, asymptotic properties, boundary-value problems, and so forth [3-5, 715], we extended representation of a solution of a system of differential equations of second order with delay [1]

$$
\ddot{x}(t)=-B^{2} x(t-\tau)
$$

to the case of two delays

$$
\ddot{x}(t)=-B_{1}^{2} x\left(t-\tau_{1}\right)-B_{2}^{2} x\left(t-\tau_{2}\right),
$$

where the linear parts were given by permutable matrices [16]. Equations (1), (2), and the below-stated (11) with $f \equiv 0$ are generalizations of the scalar equation

$$
\ddot{x}(t)=-b^{2} x(t)
$$

representing linear oscillator, to $N$-dimensional space with one or multiple fixed delays. Clearly, each solution of the latter equation is oscillating whenever $0 \neq b \in \mathbb{R}$. Analogically, (1) with $x \in \mathbb{R}^{N}$ can have at least one oscillating solution whenever $N$ is odd. Indeed, if $B$ is $N \times N$ matrix, $N \geq 3$ is odd, and $B$ has a simple real nonzero eigenvalue $\lambda$, then there exists a regular matrix $S$ such that $S^{-1} B S=J=\left(\begin{array}{ll}\lambda & 0 \\ 0 & \tilde{J}\end{array}\right)$ where $\widetilde{J}$ is $(N-1) \times(N-1)$ matrix. On letting $x=S y$, one gets

$$
\ddot{y}=-J^{2} y(t-\tau)
$$

or rewrites as the system

$$
\begin{aligned}
& \ddot{y}_{1}=-\lambda^{2} y_{1}(t-\tau), \\
& \ddot{y}_{2}=-\widetilde{J}^{2} y_{2}(t-\tau),
\end{aligned}
$$

where $y=\left(y_{1}, y_{2}\right) \in \mathbb{R} \times \mathbb{R}^{N-1}$. Note that the first column $v$ of $S$ is the eigenvector of $B$ corresponding to $\lambda$. Clearly, if solution $y_{1}$ of (5) is oscillating, then solution $y$ of (4) is oscillating in the first coordinate whenever its initial 
condition satisfies $\{y(t) \mid t \in[-\tau, 0]\} \subset \mathbb{R} \times\{0\}^{N-1}$. Consequently, solution $x$ of (1) is oscillating in $\operatorname{span}\{v\}$ whenever $\{x(t) \mid t \in[-\tau, 0]\} \subset \operatorname{span}\{v\}$. Taking $y_{1}(t)=e^{\mu t}$, one obtains characteristic equation $\mu^{2}=-\lambda^{2} e^{-\mu \tau}$ of (5), which has solutions $\mu_{1,2}=\alpha \pm \imath \beta \in \mathbb{C}$ with $\beta \neq 0$. Thus, $y_{1}$ is oscillating.

On the other hand, there can exist a nonoscillating solution of the system (1) whenever $x \in \mathbb{R}^{N}$ and $N$ is even. For instance, if $N=2$ and $B=\left(\begin{array}{rr}0 & 1 \\ -1 & 0\end{array}\right)$, then (1) has the form

$$
\ddot{x}(t)=x(t-\tau)
$$

with $x \in \mathbb{R}^{2}$, which, obviously, does not have an oscillating solution satisfying nonoscillating initial condition. Similarly, it can be shown that system with odd dimension can possess a nonoscillating solution satisfying an appropriate initial condition.

For simplicity, we call the generalizations (1), (2), and (11) with $f \equiv 0$, of scalar equation (3), oscillating although their solutions do not always have to be oscillating. Nevertheless, at the end of this paper, in Corollary 8 we state the representation of a solution of more general system (86) without squares of matrices.

We note that the delayed matrix exponential from [1-5] as well as the representation of a solution of second-order differential equations derived in $[1,16]$ and in this paper can lead to new results in nonlinear boundary value problems for impulsive functional differential equations considered in [17] or stochastic delayed differential equations from [18].

So, in the present paper, we extend our result from [16] to three and more delays by the assumption of pairwise permutable matrices defining linear parts. By such an assumption, we are able to construct matrix functions solving homogeneous system of differential equations of second order with any number of fixed delays, and, consequently, we use these functions to represent a solution of the corresponding nonhomogeneous initial value problem. As will be shown in the next sections, extending from two to more delays brings many technical difficulties, for example, the use of multinomial coefficients. Naturally, the results of the present paper hold with one or two different delays as well. However, these cases can by studied in a simpler way, which was already done in $[1,16]$. Thus, we focus our attention on the case of three and more different delays.

First, we recall our result from [16].

Theorem 1. Let $\tau_{1}, \tau_{2}>0, \tau:=\max \left\{\tau_{1}, \tau_{2}\right\}$, and $\varphi \in$ $C^{1}\left([-\tau, 0], \mathbb{R}^{N}\right)$. Let $B_{1}, B_{2}$ be $N \times N$ permutable matrices; that is, $B_{1} B_{2}=B_{2} B_{1}$, and let $f:[0, \infty) \rightarrow \mathbb{R}^{N}$ be a given function . Solution $x(t)$ of

$$
\ddot{x}(t)=-B_{1}^{2} x\left(t-\tau_{1}\right)-B_{2}^{2} x\left(t-\tau_{2}\right)+f(t)
$$

satisfying initial condition

$$
\begin{aligned}
& x(t)=\varphi(t), \\
& \dot{x}(t)=\dot{\varphi}(t), \quad-\tau \leq t \leq 0
\end{aligned}
$$

has the form

$$
x(t)= \begin{cases}\varphi(t), & -\tau \leq t<0, \\ \mathscr{X}(t) \varphi(0)+\mathcal{Y}(t) \dot{\varphi}(0) & \\ -B_{1}^{2} \int_{-\tau_{1}}^{0} \mathscr{Y}\left(t-\tau_{1}-s\right) \varphi(s) d s & \\ -B_{2}^{2} \int_{-\tau_{2}}^{0} \mathcal{Y}\left(t-\tau_{2}-s\right) \varphi(s) d s & \\ +\int_{0}^{t} \mathcal{Y}(t-s) f(s) d s, & 0 \leq t,\end{cases}
$$

where

$$
\begin{aligned}
\mathscr{X}(t)= & \mathcal{X}_{\tau_{1}, \tau_{2}}^{B_{1}^{2}, B_{2}^{2}}(t) \\
:= & \sum_{\substack{i, j \geq 0 \\
i \tau_{1}+j \tau_{2} \leq t}}(-1)^{i+j} \\
& \times\left(\begin{array}{c}
i+j \\
i
\end{array}\right) B_{1}^{2 i} B_{2}^{2 j} \frac{\left(t-i \tau_{1}-j \tau_{2}\right)^{2(i+j)}}{(2(i+j)) !}, \\
\mathcal{Y}(t)= & \mathscr{Y}_{\tau_{1}, \tau_{2}}^{B_{1}^{2}, B_{2}^{2}}(t) \\
:= & \sum_{\substack{i, j \geq 0 \\
i \tau_{1}+j \tau_{2} \leq t}}(-1)^{i+j} \\
& \times\left(\begin{array}{c}
i+j \\
i
\end{array}\right) B_{1}^{2 i} B_{2}^{2 j} \frac{\left(t-i \tau_{1}-j \tau_{2}\right)^{2(i+j)+1}}{(2(i+j)+1) !} .
\end{aligned}
$$

We will denote $\Theta$ and $E$ the $N \times N$ zero and identity matrix, respectively.

\section{Systems with Multiple Delays}

In this section, we derive the representation of a solution of

$$
\ddot{x}(t)=-B_{1}^{2} x\left(t-\tau_{1}\right)-\cdots-B_{n}^{2} x\left(t-\tau_{n}\right)+f(t)
$$

satisfying the initial condition (8), where $n \geq 3, \tau_{1}, \ldots, \tau_{n}>0$, $\tau:=\max _{i=1, \ldots, n} \tau_{i}, B_{1}, \ldots, B_{n}$ are $N \times N$ pairwise permutable matrices; that is, $B_{i} B_{j}=B_{j} B_{i}$ for each $i, j \in\{1, \ldots, n\}, \varphi \in$ $C^{1}\left([-\tau, 0], \mathbb{R}^{N}\right)$, and $f:[0, \infty) \rightarrow \mathbb{R}^{N}$ are given functions. The solution $x(t)$ will be represented using matrix functions analogical to (10) and will be stated in Section 3. We note that the same problems with $n=1,2$ were studied in $[1,16]$.

From now on, we assume the property of empty sum and empty product; that is,

$$
\begin{array}{lll}
\sum_{i \in \emptyset} f(i)=0, & & \sum_{i \in \emptyset} F(i)=\Theta, \\
\prod_{i \in \emptyset} f(i)=1, & & \prod_{i \in \emptyset} F(i)=E
\end{array}
$$

for any function $f$ and matrix function $F$, whether they are defined or not for indicated argument. 
We recall that $\left(j_{1}, \ldots, j_{n}\right)$ ! is a multinomial coefficient [19] given by

$$
\left(j_{1}, \ldots, j_{n}\right) !=\frac{\left(j_{1}+\cdots+j_{n}\right) !}{j_{1} ! \cdots j_{n} !} .
$$

Note that if $n=2$, then $\left(j_{1}, j_{2}\right)=\left(\begin{array}{c}j_{1}+j_{2} \\ j_{1}\end{array}\right)$ and (20) coincides with (10).

We will need a property of multinomial coefficients described in the next lemma.

Lemma 2. Let $n \geq 2$ be fixed. Then

$$
\begin{aligned}
\left(i_{1}, i_{2}, \ldots, i_{n}\right) != & \left(i_{1}-1, i_{2}, \ldots, i_{n}\right) ! \\
& +\left(i_{1}, i_{2}-1, i_{3}, \ldots, i_{n}\right) !+\left(i_{1}, \ldots, i_{n-1}, i_{n}-1\right) !
\end{aligned}
$$

for any $i_{1}, \ldots, i_{n} \geq 1$.

Proof. If $n=2$, then the statement follows from the property of binomial coefficients:

$$
\begin{aligned}
\left(i_{1}, i_{2}\right) ! & =\left(\begin{array}{c}
i_{1}+i_{2} \\
i_{1}
\end{array}\right)=\left(\begin{array}{c}
i_{1}+i_{2}-1 \\
i_{1}-1
\end{array}\right)+\left(\begin{array}{c}
i_{1}+i_{2}-1 \\
i_{1}
\end{array}\right) \\
& =\left(i_{1}-1, i_{2}\right) !+\left(i_{1}, i_{2}-1\right) ! .
\end{aligned}
$$

Let the statement be true for $n-1$. Next, we use the property of multinomial coefficient

$$
\left(i_{1}, i_{2}, i_{3}, \ldots, i_{n}\right) !=\left(i_{1}+i_{2}, i_{3}, \ldots, i_{n}\right) !\left(i_{1}, i_{2}\right) !
$$

with inductive hypothesis to derive

$$
\begin{aligned}
\left(i_{1}, i_{2}, i_{3}, \ldots, i_{n}\right) ! & \\
= & \left(i_{1}+i_{2}-1, i_{3}, \ldots, i_{n}\right) !+\left(i_{1}+i_{2}, i_{3}-1, \ldots, i_{n}\right) ! \\
& \left.+\cdots+\left(i_{1}+i_{2}, i_{3}, \ldots, i_{n}-1\right) !\right]\left(i_{1}, i_{2}\right) !
\end{aligned}
$$

Clearly, from (16), we get

$$
\begin{gathered}
\left(i_{1}+i_{2}, i_{3}-1, \ldots, i_{n}\right) !\left(i_{1}, i_{2}\right) !=\left(i_{1}, i_{2}, i_{3}-1, \ldots, i_{n}\right) !, \\
\vdots \\
\left(i_{1}+i_{2}, i_{3}, \ldots, i_{n}-1\right) !\left(i_{1}, i_{2}\right) !=\left(i_{1}, i_{2}, i_{3}, \ldots, i_{n}-1\right) ! .
\end{gathered}
$$

Applying the case $n=2$ (property of binomial coefficient) and (16), we get

$$
\begin{aligned}
\left(i_{1}+\right. & \left.i_{2}-1, i_{3}, \ldots, i_{n}\right) !\left(i_{1}, i_{2}\right) ! \\
& =\left(i_{1}+i_{2}-1, i_{3}, \ldots, i_{n}\right) !\left[\left(i_{1}-1, i_{2}\right) !+\left(i_{1}, i_{2}-1\right) !\right] \\
& =\left(i_{1}-1, i_{2}, i_{3}, \ldots, i_{n}\right) !+\left(i_{1}, i_{2}-1, i_{3}, \ldots, i_{n}\right) !
\end{aligned}
$$

Putting (18) and (19) in (17), we obtain that the statement holds for $n$ and the proof is complete.
In further work, we write $(\{j \mid j \in M\})$ ! for the multinomial coefficient of elements of the finite set $M$, and $(i,\{j \mid j \in M\})$ ! for the multinomial coefficient of $i$ and elements of the finite set $M$; for example, if $M=\{1,2\}$, then $(a,\{j \mid j \in M\}) !=(a, 1,2) !$. For the completeness, we define $(\{j \mid j \in \emptyset\}) !:=1$.

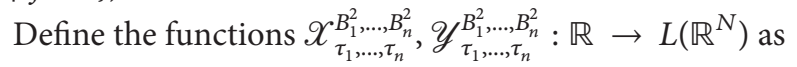

$$
\begin{aligned}
X_{\tau_{1}, \ldots, \tau_{n}}^{B_{1}^{2}, \ldots, B_{n}^{2}}(t) & =\sum_{\substack{j_{1}, \ldots, j_{n} \geq 0 \\
j_{1} \tau_{1}+\cdots+j_{n} \tau_{n} \leq t}}(-1)^{j_{1}+\cdots+j_{n}}\left(j_{1}, \ldots, j_{n}\right) ! \\
& \times \prod_{i=1}^{n} B_{i}^{2 j_{i}} \frac{\left(t-j_{1} \tau_{1}-\cdots-j_{n} \tau_{n}\right)^{2\left(j_{1}+\cdots+j_{n}\right)}}{\left(2\left(j_{1}+\cdots+j_{n}\right)\right) !}, \\
\mathcal{Y}_{\tau_{1}, \ldots, \tau_{n}}^{B_{1}^{2}, \ldots, B_{n}^{2}}(t) & :=\sum_{\substack{j_{1}, \ldots, j_{n} \geq 0 \\
j_{1} \tau_{1}+\cdots+j_{n} \tau_{n} \leq t}}(-1)^{j_{1}+\cdots+j_{n}}\left(j_{1}, \ldots, j_{n}\right) ! \\
& \times \prod_{i=1}^{n} B_{i}^{2 j_{i}} \frac{\left(t-j_{1} \tau_{1}-\cdots-j_{n} \tau_{n}\right)^{2\left(j_{1}+\cdots+j_{n}\right)+1}}{\left(2\left(j_{1}+\cdots+j_{n}\right)+1\right) !}
\end{aligned}
$$

for any $t \in \mathbb{R}$.

We will need functions $\mathscr{X}_{\tau}^{B^{2}}, \mathscr{Y}_{\tau}^{B^{2}}: \mathbb{R} \rightarrow L\left(\mathbb{R}^{N}\right)$ for $\tau>0$ and $N \times N$ complex matrix $B$ (cf. [16]) defined as

$$
\begin{aligned}
X_{\tau}^{B^{2}}(t) & :=\sum_{\substack{i \geq 0 \\
i \tau \leq t}}(-1)^{i} B^{2 i} \frac{(t-i \tau)^{2 i}}{(2 i) !}, \\
\mathscr{Y}_{\tau}^{B^{2}}(t) & :=\sum_{\substack{i \geq 0 \\
i \tau \leq t}}(-1)^{i} B^{2 i} \frac{(t-i \tau)^{2 i+1}}{(2 i+1) !}
\end{aligned}
$$

with the properties

$$
\begin{gathered}
\dot{X}_{\tau}^{B^{2}}(t)=-B^{2} \mathscr{Y}_{\tau}^{B^{2}}(t-\tau), \quad \ddot{X}_{\tau}^{B^{2}}(t)=-B^{2} \mathscr{X}_{\tau}^{B^{2}}(t-\tau), \\
\dot{y}_{\tau}^{B^{2}}(t)=\mathscr{X}_{\tau}^{B^{2}}(t), \quad \ddot{\mathscr{Y}}_{\tau}^{B^{2}}(t)=-B^{2} \mathscr{Y}_{\tau}^{B^{2}}(t-\tau)
\end{gathered}
$$

for any $t \in \mathbb{R}$, considering the one-sided derivatives at $-\tau, 0$.

Some of properties of functions $\mathscr{X}_{\tau_{1}, \ldots, \tau_{n}}^{B_{1}^{2}, \ldots, B_{n}^{2}}$ and $\mathscr{Y}_{\tau_{1}, \ldots, \tau_{n}}^{B_{1}^{2}, \ldots B_{n}^{2}}$ are concluded in Lemma 4, but to prove it we will need the next lemma.

Lemma 3. Let $n \geq 1$ and $\tau_{1}, \ldots, \tau_{n}>0$. Let $B_{1}, \ldots, B_{n}$ be $N \times N$ pairwise permutable matrices, that is, $B_{i} B_{j}=B_{j} B_{i}$ for each $i, j \in\{1, \ldots, n\}$. Then for any $t \in \mathbb{R}$,

$$
\begin{aligned}
& x_{\tau_{1}, \ldots, \tau_{n}}^{B_{1}^{2}, \ldots, B_{n}^{2}}(t)=\sum_{M \subset\{1, \ldots, n\}} S_{M}(t), \\
& \mathcal{Y}_{\tau_{1}, \ldots, \tau_{n}}^{B_{1}^{2}, \ldots B_{n}^{2}}(t)=\sum_{M \subset\{1, \ldots, n\}} \widetilde{S}_{M}(t),
\end{aligned}
$$


where the sums are taken over all subsets of $\{1, \ldots, n\}$ including the trivial ones, and

$$
\begin{aligned}
S_{M}(t):= & \sum_{\substack{j_{i} \geq 1, i \in M \\
\sum_{i \in M} j_{i} \tau_{i} \leq t}}(-1)^{\sum_{i \in M} j_{i}}\left(\left\{j_{i} \mid i \in M\right\}\right) ! \\
& \times \prod_{i \in M} B_{i}^{2 j_{i}} \frac{\left(t-\sum_{i \in M} j_{i} \tau_{i}\right)^{2 \sum_{i \in M} j_{i}}}{\left(2 \sum_{i \in M} j_{i}\right) !}, \\
\widetilde{S}_{M}(t):= & \sum_{j_{i} \geq 1, i \in M}(-1)^{\sum_{i \in M} j_{i}}\left(\left\{j_{i} \mid i \in M\right\}\right) ! \\
& \times \prod_{i \in M} B_{i}^{2 j_{i}} \frac{\left(t-\sum_{i \in M} j_{i} \tau_{i}\right)^{2 \sum_{i \in M} j_{i}+1}}{\left(2 \sum_{i \in M} j_{i}+1\right) !} .
\end{aligned}
$$

Proof. Denote $\mathbb{N}_{0}, \mathbb{N}$ the set of all nonnegative, positive integers, respectively; that is, $\mathbb{N}_{0}=\{0\} \cup \mathbb{N}$. Thus, we have the trivial identity

$$
\begin{aligned}
& \underbrace{\mathbb{N}_{0} \times \cdots \times \mathbb{N}_{0}}_{n} \\
& =(\{0\} \times \underbrace{\mathbb{N}_{0} \times \cdots \times \mathbb{N}_{0}}_{n-1}) \cup(\mathbb{N} \times \underbrace{\mathbb{N}_{0} \times \cdots \times \mathbb{N}_{0}}_{n-1}) \\
& =\cdots=\bigcup_{M_{1}, \ldots, M_{n} \in\{\{0\}, \mathbb{N}\}} M_{1} \times \cdots \times M_{n} .
\end{aligned}
$$

Analogically, for any $t \in \mathbb{R}$ each $n$-tuple $j_{1}, \ldots, j_{n} \geq 0$ such that $\sum_{i=1}^{n} j_{i} \tau_{i} \leq t$ can be divided in two distinct sets of $i$-s so that $j_{i} \geq 1$ if $i \in M \subset\{1, \ldots, n\}$ and $j_{i}=0$ if $i \in$ $\{1, \ldots, n\} \backslash M$. That is, $M$ denotes the set of all indices $i$ such that $j_{i}=0$. Moreover, $\sum_{i=1}^{n} j_{i} \tau_{i}=\sum_{i \in M} j_{i} \tau_{i}$. Accordingly, we can write

$$
\begin{aligned}
& \left\{\left(j_{1}, \ldots, j_{n}\right) \in \mathbb{N}_{0}^{n} \mid \sum_{i=1}^{n} j_{i} \tau_{i} \leq t\right\} \\
& =\bigcup_{M \subset\{1, \ldots, n\}}\left\{\left(j_{1}, \ldots, j_{n}\right) \in \mathbb{N}_{0}^{n} \mid\right. \\
& \left.j_{i}=0 \forall i \notin M, \sum_{i \in M} j_{i} \tau_{i} \leq t\right\},
\end{aligned}
$$

where the union is taken over all subsets of $\{1, \ldots, n\}$ including the trivial ones. So, in the view of definition (20), the statement for $\mathscr{X}_{\tau_{1}, \ldots, \tau_{n}}^{B_{1}^{2}, \ldots, B_{n}^{2}}$ follows.

Statement for $\mathcal{Y}_{\tau_{1}, \ldots, \tau_{n}}^{B_{1}^{2}, \ldots, B_{n}^{2}}$ can be proved in a similar way.

Lemma 4. Let $n \geq 3$ and $\tau_{1}, \ldots, \tau_{n}>0$. Let $B_{1}, \ldots, B_{n}$ be $N \times N$ pairwise permutable matrices; that is, $B_{i} B_{j}=B_{j} B_{i}$ for each $i, j \in\{1, \ldots, n\}$. Then the following holds for any $t \in \mathbb{R}$ :
(1) if $B_{i}=\Theta$ for some $i \in\{1, \ldots, n\}$, then

$$
\mathscr{X}_{\tau_{1}, \ldots, \tau_{i-1}, \tau_{i}, \tau_{i+1}, \ldots, \tau_{n}}^{B_{1}^{2}, \ldots, B_{n}^{2}}(t)=\mathscr{X}_{\tau_{1}, \ldots, \tau_{i-1}, \tau_{i+1}, \ldots, \tau_{n}}^{B_{1}^{2}, \ldots, B_{n}^{2}}(t),
$$

(2) if $\tau_{i}=\tau_{k}$ for $i<k, i, k \in\{1, \ldots, n\}$, then

$$
\begin{aligned}
& \mathcal{X}_{\tau_{1}, \ldots, \tau_{i-1}, \tau_{i}, \tau_{i+1}, \ldots, \tau_{k-1}, \tau_{k}, \tau_{k+1}, \ldots, \tau_{n}}^{B_{1}^{2}, \ldots, B_{n}^{2}}(t) \\
& =\mathscr{X}_{\tau_{1}, \ldots, \tau_{i-1}, \tau_{i}, \tau_{i+1}, \ldots, \tau_{k-1}, \tau_{k+1}, \ldots, \tau_{n}}^{B_{1}^{2}, \ldots, B_{i-1}^{2}, B_{n}^{2}+B_{k}^{2}, B_{i+1}^{2}, \ldots, B_{k-1}^{2}, B_{n}^{2}}(t),
\end{aligned}
$$

(3) for any bijective mapping $\sigma:\{1, \ldots, n\} \rightarrow\{1, \ldots, n\}$ we get

$$
\mathscr{X}_{\tau_{1}, \ldots, \tau_{n}}^{B_{1}^{2}, \ldots, B_{n}^{2}}(t)=\mathscr{X}_{\tau_{\sigma(1)}, \ldots, \tau_{\sigma(n)}}^{B_{\sigma(1)}^{2}, \ldots, B_{\sigma(n)}^{2}}(t),
$$

(4) taking the one-sided derivatives at $0, \tau_{1}, \ldots, \tau_{n}$, then

$$
\begin{aligned}
\ddot{X}_{\tau_{1}, \ldots, \tau_{n}}^{B_{1}^{2}, \ldots, B_{n}^{2}}(t)= & -B_{1}^{2} \mathscr{X}_{\tau_{1}, \ldots, \tau_{n}}^{B_{1}^{2}, \ldots, B_{n}^{2}}\left(t-\tau_{1}\right) \\
& -\cdots-B_{n}^{2} \mathscr{X}_{\tau_{1}, \ldots, \tau_{n}}^{B_{1}^{2}, \ldots, B_{n}^{2}}\left(t-\tau_{n}\right),
\end{aligned}
$$

(5) considering the one-sided derivatives at 0 (they both equal $\Theta)$, then

$$
\dot{\mathscr{Y}}_{\tau_{1}, \ldots, \tau_{n}}^{B_{1}^{2}, \ldots, B_{n}^{2}}(t)=\mathscr{X}_{\tau_{1}, \ldots, \tau_{n}}^{B_{1}^{2}, \ldots, B_{n}^{2}}(t)
$$

Statements (1)-(4) hold with $\mathcal{Y}$ instead of $\mathscr{X}$.

Proof. Statement (1) follows easily from definition of $\mathscr{X}_{\tau_{1}, \ldots, \tau_{n}}^{B_{1}^{2}, \ldots, B_{n}^{2}}$, because $\Theta^{2 i}=E$ if $i=0$ and $\Theta^{2 i}=\Theta$ whenever $i>0$. Next, if $\tau_{i}=\tau_{k}$, then

$$
\begin{aligned}
& \sum_{\substack{j_{1}, \ldots, j_{n} \geq 0 \\
j_{1} \tau_{1}+\cdots+j_{n} \tau_{n} \leq t}} F\left(j_{1}, \ldots, j_{n}\right) \\
& =\sum_{\substack{j_{1}, \ldots, j_{i-1}, l, j_{i+1}, \ldots, j_{k-1}, j_{k+1}, \ldots, j_{n} \geq 0 \\
j_{1} \tau_{1}+\cdots+j_{1-1} \tau_{i-1}+l \tau_{i}+j_{i+1} \\
+\cdots+j_{i+1} \\
+\cdots+j_{k-1} \tau_{k-1}+j_{k+1} \tau_{k+1}+\cdots+j_{n} \tau_{n} \leq t}} \sum_{\substack{j_{i}, j_{k} \geq 0 \\
j_{i}+j_{k}=l}} F\left(j_{1}, \ldots, j_{n}\right)
\end{aligned}
$$

for any matrix function $F$. Thus, using the property of multinomial coefficient (see (16))

$$
\begin{aligned}
& \left(j_{1}, \ldots, j_{n}\right) ! \\
& \quad=\left(j_{1}, \ldots, j_{i-1}, j_{i}+j_{k}, j_{i+1}, \ldots, j_{k-1}, j_{k+1}, \ldots, j_{n}\right) !\left(j_{i}, j_{k}\right) !,
\end{aligned}
$$


for (2), we obtain

$$
\begin{aligned}
& \mathscr{X}_{\tau_{1}, \ldots, \tau_{n}}^{B_{1}^{2}, \ldots, B_{n}^{2}}(t) \\
& \begin{array}{l}
=\sum_{\substack{j_{1}, \ldots, j_{i-1}, l, j_{i+1}, \ldots, j_{k-1}, j_{k+1}, \ldots, j_{n} \geq 0 \\
j_{1} \tau_{1}+\cdots+j_{i-1} \tau_{i-1}+l \tau_{i}+j_{i+1} \tau_{i+1} \\
+\cdots+j_{k-1} \tau_{k-1}+j_{k+1} \tau_{k+1}+\cdots+j_{n} \tau_{n} \leq t}}(-1)^{\sum_{s \in\{1, \ldots, n\}} j_{s}+l} \begin{array}{l}
s \neq i, k \\
\end{array} \\
\quad \times\left(j_{1}, \ldots, j_{i-1}, l, j_{i+1}, \ldots, j_{k-1}, j_{k+1}, \ldots, j_{n}\right)
\end{array} \\
& \times\left(\sum_{\substack{j_{i}, j_{k} \geq 0 \\
j_{i}+j_{k}=l}}\left(j_{i}, j_{k}\right) ! B_{i}^{2 j_{i}} B_{k}^{2 j_{k}}\right) \\
& \times \prod_{\substack{s \in\{1, \ldots, n\} \\
s \neq i, k}} B_{s}^{2 j_{s}} \frac{\left(t-\sum_{\substack{s \in\{1, \ldots, n\} \\
s \neq i, k}} \sum j_{s} \tau_{s}-l \tau_{i}\right)^{2\left(\sum_{s \in\{1, \ldots, n\}} j_{s}+l\right)}}{(2 \neq i, k}
\end{aligned}
$$

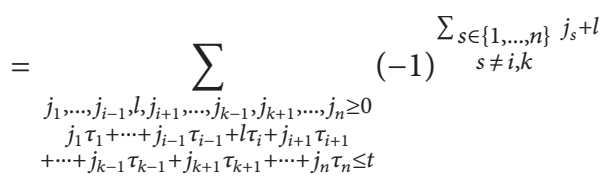

$$
\begin{aligned}
& \times\left(j_{1}, \ldots, j_{i-1}, l, j_{i+1}, \ldots, j_{k-1}, j_{k+1}, \ldots, j_{n}\right) !\left(B_{i}^{2}+B_{k}^{2}\right)^{l} \\
& \times \prod_{\substack{s \in\{1, \ldots, n\} \\
s \neq i, k}} B_{s}^{2 j_{s}} \frac{\left(t-\sum_{\substack{s \in\{1, \ldots, n\} \\
s \neq i, k}} \sum j_{s} \tau_{s}-l \tau_{i}\right)^{2\left(\sum_{s \in\{1, \ldots, n\}} j_{s}+l\right)}}{\left(2\left(\sum_{\substack{s \in\{1, \ldots, n\} \\
s \neq i, k}} \sum j_{s}+l\right)\right) !} \\
& =\mathscr{X}_{\tau_{1}, \ldots, \tau_{i-1}, \tau_{i}, \tau_{i+1}, \ldots, \tau_{k-1}, \tau_{k+1}, \ldots, \tau_{n}}^{B_{1}^{2}, \ldots, B_{i}^{2}, B_{i}^{2}+B_{k}^{2}, B_{i+1}^{2}, \ldots, B_{k}^{2}, B_{k}^{2}}(t) .
\end{aligned}
$$

Property (3) is trivial.

Now, we prove the statement (4). If $\tau:=\tau_{1}=\cdots=\tau_{n}$, then

$$
\begin{aligned}
\ddot{\mathscr{X}}_{\tau_{1}, \ldots, \tau_{n}}^{B_{1}^{2}, \ldots, B_{n}^{2}}(t)= & \ddot{X}_{\tau}^{B_{1}^{2}+\cdots+B_{n}^{2}}(t) \\
= & -\left(B_{1}^{2}+\cdots+B_{n}^{2}\right) \mathscr{X}_{\tau}^{B_{1}^{2}+\cdots+B_{n}^{2}}(t-\tau) \\
= & -B_{1}^{2} \mathscr{X}_{\tau_{1}, \ldots, \tau_{n}}^{B_{1}^{2}, \ldots, B_{n}^{2}}\left(t-\tau_{1}\right) \\
& -\cdots-B_{n}^{2} \mathscr{X}_{\tau_{1}, \ldots, \tau_{n}}^{B_{1}^{2}, \ldots, B_{n}^{2}}\left(t-\tau_{n}\right)
\end{aligned}
$$

by (2) and from the property of $\mathscr{X}_{\tau}^{B_{1}^{2}+\cdots+B_{n}^{2}}(t)$ (see (22)).

Hence, without any loss of generality, we assume that $\tau_{i} \neq \tau_{j}$ for each $i \neq j, i, j \in\{1, \ldots, n\}$ (in the other case, we collect matrices as stated in (2)). Note the case $n=2$ was proved in [16, Lemma 2.3.] Now, assume that $\mathscr{X}_{\tau_{1}, \ldots, \tau_{n-1}}^{B_{1}^{2}, \ldots, B_{n-1}^{2}}(t)$ solves

$$
\ddot{x}(t)=-B_{1}^{2} x\left(t-\tau_{1}\right)-\cdots-B_{n-1}^{2} x\left(t-\tau_{n-1}\right),
$$

that is, that the statement is fulfilled for $n-1$ different delays.
Let $\tau_{k}:=\max _{i=1, \ldots, n} \tau_{i}$. If $t<\tau_{k}$, then $t-\tau_{k}<0$, that is,

$$
\mathscr{X}_{\tau_{1}, \ldots, \tau_{n}}^{B_{1}^{2}, \ldots, B_{n}^{2}}\left(t-\tau_{k}\right)=\Theta
$$

and from definition (20) it holds

$$
\mathscr{X}_{\tau_{1}, \ldots, \tau_{n}}^{B_{1}^{2}, \ldots, B_{n}^{2}}(t)=\mathscr{X}_{\tau_{1}, \ldots, \tau_{k-1}, \tau_{k+1}, \ldots, \tau_{n}}^{B_{1}^{2}, \ldots, B_{k}^{2}, B_{k}^{2}}(t)
$$

for such $t$. Consequently,

$$
\begin{aligned}
\ddot{\mathscr{X}}_{\tau_{1}, \ldots, \tau_{n}}^{B_{1}^{2}, \ldots, B_{n}^{2}}(t) & =\ddot{\mathscr{X}}_{\tau_{1}, \ldots, \tau_{k-1}, \tau_{k+1}, \ldots, \tau_{n}}^{B_{1}^{2}, \ldots, B_{k-1}^{2}, B_{k+1}^{2}, \ldots, B_{n}^{2}}(t) \\
& =-\sum_{\substack{i=1, \ldots, n \\
i \neq k}} B_{i}^{2} \mathscr{X}_{\tau_{1}, \ldots, \tau_{k-1}, \tau_{k+1}, \ldots, \tau_{n}}^{B_{1}^{2}, \ldots, B_{n}^{2}}\left(t-\tau_{i}\right) \\
& =-\sum_{i=1}^{n} B_{i}^{2} \mathscr{X}_{\tau_{1}, \ldots, \tau_{n}}^{B_{1}^{2}, \ldots, B_{n}^{2}}\left(t-\tau_{i}\right)
\end{aligned}
$$

by the inductive hypothesis.

Now, let $t \geq \max _{i=1, \ldots, n} \tau_{i}$. Applying Lemma 3, we get

$$
\mathscr{X}_{\tau_{1}, \ldots, \tau_{n}}^{B_{1}^{2}, \ldots, B_{n}^{2}}(t)=\sum_{M \subset\{1, \ldots, n\}} S_{M}(t)
$$

with $S_{M}(t)$ given by (24) and the sum taken over all subsets of $\{1, \ldots, n\}$ including the trivial ones. Note that

$$
\begin{aligned}
S_{\emptyset}(t) & =\sum_{\substack{j_{i} \geq 1, i \in \emptyset \\
0 \leq t}}(-1)^{0}\left(\left\{j_{i} \mid i \in \emptyset\right\}\right) ! E \frac{(t-0)^{0}}{0 !} \\
& =\sum_{0 \leq t} E=E \chi_{[0, \infty)}(t)
\end{aligned}
$$

with a characteristic function $\chi_{\widetilde{M}}$ of a set $\widetilde{M}$ given by

$$
\chi_{\widetilde{M}}(t)= \begin{cases}1, & t \in \widetilde{M}, \\ 0, & t \notin \widetilde{M} .\end{cases}
$$

Since each $M \subset\{1, \ldots, n\}$ is a finite set, Lemma 2 yields

$$
\left(\left\{j_{i} \mid i \in M\right\}\right) !=\sum_{i \in M}\left(j_{i}-1,\left\{j_{k} \mid k \in M \backslash\{i\}\right\}\right) ! .
$$

We apply this identity to derive a formula for the second derivative of $S_{M}$ for any $\emptyset \neq M \subset\{1, \ldots, n\}$ :

$$
\begin{aligned}
S_{M}^{\prime \prime}( & t) \\
= & \sum_{\substack{j_{i} \geq 1, i \in M \\
\sum_{i \in M} j_{i} \tau_{i} \leq t}}(-1)^{\sum_{i \in M} j_{i}}\left(\left\{j_{i} \mid i \in M\right\}\right) ! \\
& \times \prod_{i \in M} B_{i}^{2 j_{i}} \frac{\left(t-\sum_{i \in M} j_{i} \tau_{i}\right)^{2\left(\sum_{i \in M} j_{i}-1\right)}}{\left(2\left(\sum_{i \in M} j_{i}-1\right)\right) !} \\
= & \sum_{i \in M} \sum_{\sum_{k \in M} j_{k} \geq 1, k \in M}(-1)^{\sum_{k \in M} j_{k}}\left(j_{i}-1,\left\{j_{k} \mid k \in M \backslash\{i\}\right\}\right) ! \\
& \times \prod_{k \in M} B_{k}^{2 j_{k}} \frac{\left(t-\tau_{i}-\sum_{k \in M \backslash\{i\}} j_{k} \tau_{k}-\left(j_{i}-1\right) \tau_{i}\right)^{2\left(\sum_{k \in M} j_{k}-1\right)}}{\left(2\left(\sum_{k \in M} j_{k}-1\right)\right) !} .
\end{aligned}
$$


Next, for any fixed $i \in\{1, \ldots, n\}$ we split the second sum to $j_{i}=1$ and $j_{i} \geq 2$, that is,

$$
\begin{aligned}
& \sum_{\substack{j_{k} \geq 1, k \in M \\
\sum_{k \in M} j_{k} \tau_{k} \leq t}} F\left(j_{1}, \ldots, j_{i-1}, j_{i}, j_{i+1}, \ldots, j_{n}\right) \\
& =\sum_{\substack{j_{k} \geq 1, k \in M \backslash\{i\} \\
\sum_{k \in M \backslash i\}} j_{k} \tau_{k} \leq t-\tau_{i}}} F\left(j_{1}, \ldots, j_{i-1}, 1, j_{i+1}, \ldots, j_{n}\right) \\
& \quad+\sum_{\substack{j_{k} \geq 1, k \in M \backslash\{i\} \\
j_{i} \geq 2}} F\left(j_{1}, \ldots, j_{i-1}, j_{i}, j_{i+1}, \ldots, j_{n}\right),
\end{aligned}
$$

and use the equality

$$
\begin{aligned}
& \sum_{\substack{j_{k} \geq 1, k \in M \backslash\{i\} \\
j_{i} \geq 2 \\
\sum_{k \in M} j_{k} \tau_{k} \leq t}} F\left(j_{1}, \ldots, j_{i-1}, j_{i}, j_{i+1}, \ldots, j_{n}\right) \\
& \quad=\sum_{\substack{j_{k} \geq 1, k \in M \\
\sum_{k \in M} j_{k} \tau_{k} \leq t-\tau_{i}}} F\left(j_{1}, \ldots, j_{i-1}, j_{i}+1, j_{i+1}, \ldots, j_{n}\right)
\end{aligned}
$$

since

$$
\sum_{k \in M} j_{k} \tau_{k} \leq t \Longleftrightarrow \sum_{k \in M \backslash\{i\}} j_{k} \tau_{k}+\left(j_{i}-1\right) \tau_{i} \leq t-\tau_{i} .
$$

So we obtain

$$
S_{M}^{\prime \prime}(t)=-\sum_{i \in M} B_{i}^{2}\left(S_{M \backslash\{i\}}\left(t-\tau_{i}\right)+S_{M}\left(t-\tau_{i}\right)\right)
$$

for each $\emptyset \neq M \quad \subset\{1, \ldots, n\}$. Obviously, $S_{\emptyset}^{\prime \prime}(t)=\Theta$. Consequently,

$$
\begin{aligned}
\ddot{\mathscr{X}}_{\tau_{1}, \ldots, \tau_{n}}^{B_{1}^{2}, \ldots, B_{n}^{2}}(t) & \\
= & -\sum_{\emptyset \neq M \subset\{1, \ldots, n\}} \sum_{i \in M} B_{i}^{2}\left(S_{M \backslash\{i\}}\left(t-\tau_{i}\right)+S_{M}\left(t-\tau_{i}\right)\right) \\
= & -\sum_{\emptyset \neq M \subset\{1, \ldots, n\}} \sum_{i \in M} B_{i}^{2} S_{M \backslash\{i\}}\left(t-\tau_{i}\right) \\
& -\sum_{\emptyset \neq M \subset\{1, \ldots, n\}} \sum_{i \in M} B_{i}^{2} S_{M}\left(t-\tau_{i}\right) .
\end{aligned}
$$

Now, we add and subtract

$$
\sum_{\emptyset \neq M \subset\{1, \ldots, n\}} \sum_{i \notin M} B_{i}^{2} S_{M}\left(t-\tau_{i}\right)
$$

to the right-hand side of (50) to get

$$
\begin{aligned}
& \ddot{X}_{\tau_{1}, \ldots, \tau_{n}}^{B_{1}^{2}, \ldots, B_{n}^{2}}(t) \\
& =-\sum_{\emptyset \neq M \subset\{1, \ldots, n\}} \sum_{i=1}^{n} B_{i}^{2} S_{M}\left(t-\tau_{i}\right) \\
& \quad+\sum_{\emptyset \neq M \subset\{1, \ldots, n\}} \sum_{i \notin M} B_{i}^{2} S_{M}\left(t-\tau_{i}\right) \\
& \quad-\sum_{\emptyset \neq M \subset\{1, \ldots, n\}} \sum_{i \in M} B_{i}^{2} S_{M \backslash\{i\}}\left(t-\tau_{i}\right)
\end{aligned}
$$

and apply $M=M \backslash\{i\}$ whenever $i \notin M$ :

$$
\begin{aligned}
\ddot{X}_{\tau_{1}, \ldots, \tau_{n}}^{B_{1}^{2}, \ldots B_{n}^{2}}(t) & \\
= & -\sum_{\emptyset \neq M \subset\{1, \ldots, n\}} \sum_{i=1}^{n} B_{i}^{2} S_{M}\left(t-\tau_{i}\right) \\
& +\sum_{\emptyset \neq M \subset\{1, \ldots, n\}} \sum_{i \notin M} B_{i}^{2} S_{M \backslash\{i\}}\left(t-\tau_{i}\right) \\
& -\sum_{\emptyset \neq M \subset\{1, \ldots, n\}} \sum_{i \in M} B_{i}^{2} S_{M \backslash\{i\}}\left(t-\tau_{i}\right) .
\end{aligned}
$$

Denoting \# $M$ the number of elements of the set $M$, we split the last two terms of the right-hand side of the latter equality with respect to

$$
\begin{aligned}
\sum_{\emptyset \neq M \subset\{1, \ldots, n\}}=\sum_{\substack{M \subset\{1, \ldots, n\} \\
1 \leq \# M \leq n-1}}+\sum_{\substack{M \subset\{1, \ldots, n\} \\
\# M=n}} \text {. } \sum_{\substack{M \subset\{1, \ldots, n\} \\
\# M=1}}+\sum_{\substack{M \subset\{1, \ldots, n\} \\
2 \leq \# M \leq n}} .
\end{aligned}
$$

Hence, we have

$$
\begin{aligned}
\sum_{\emptyset \neq M \subset\{1, \ldots, n\}} & \sum_{\substack{i \notin M \\
\emptyset \neq M \subset\{1, \ldots, n\}}} B_{i}^{2} S_{M \backslash\{i\}}\left(t-\tau_{i}\right) \\
& -\sum_{i \in M} B_{i}^{2} S_{M \backslash\{i\}}\left(t-\tau_{i}\right) \\
= & \sum_{\substack{M \subset\{1, \ldots, n\} \\
1 \leq \# M \leq n-1}} \sum_{i \notin M} B_{i}^{2} S_{M \backslash\{i\}}\left(t-\tau_{i}\right) \\
& +\sum_{\substack{M=\{1, \ldots, n\} \\
i \notin M}} \sum_{i \neq 1} B_{i}^{2} S_{M \backslash\{i\}}\left(t-\tau_{i}\right) \\
& -\sum_{\substack{M \in\{\{1\}, \ldots,\{n\}\} \\
i \in M}} \sum_{i}^{2} S_{M \backslash\{i\}}\left(t-\tau_{i}\right) \\
& -\sum_{\substack{M \subset\{1, \ldots, n\} \\
2 \leq \# M \leq n}} \sum_{i \in M} B_{i}^{2} S_{M \backslash\{i\}}\left(t-\tau_{i}\right) .
\end{aligned}
$$

Now, we show that

$$
\begin{aligned}
\sum_{\substack{M \subset\{1, \ldots, n\} \\
1 \leq \# M \leq n-1}} \sum_{i \notin M} B_{i}^{2} S_{M \backslash\{i\}}\left(t-\tau_{i}\right) \\
=\sum_{\substack{M \subset\{1, \ldots, n\} \\
2 \leq \# M \leq n}} \sum_{i \in M} B_{i}^{2} S_{M \backslash\{i\}}\left(t-\tau_{i}\right) .
\end{aligned}
$$

Let $M \subset\{1, \ldots, n\}$, and let $i \notin M$ be arbitrary and fixed such that $1 \leq \# M \leq n-1$. Then, clearly,

$$
B_{i} S_{M \backslash\{i\}}\left(t-\tau_{i}\right)=B_{i} S_{(M \cup\{i\}) \backslash\{i\}}\left(t-\tau_{i}\right)
$$

and $2 \leq \#(M \cup\{i\}) \leq n, i \in M \cup\{i\}$. Moreover, if $M_{1}, M_{2} \subset$ $\{1, \ldots, n\}, i \notin M_{1,2}$ are such that $M_{1} \neq M_{2}, 1 \leq \# M_{1,2} \leq n-1$, then $M_{1} \cup\{i\} \neq M_{2} \cup\{i\}$. 
On the other side, if $M \subset\{1, \ldots, n\}, i \in M$ are arbitrary and fixed such that $2 \leq \# M \leq n$, then

$$
B_{i} S_{M \backslash\{i\}}\left(t-\tau_{i}\right)=B_{i} S_{(M \backslash\{i\}) \backslash\{i\}}\left(t-\tau_{i}\right)
$$

and $1 \leq \#(M \backslash\{i\}) \leq n-1, i \notin M \backslash\{i\}$. Furthermore, if $M_{1}, M_{2} \subset\{1, \ldots, n\}, i \in M_{1,2}$ are such that $M_{1} \neq M_{2}, 2 \leq$ $\# M_{1,2} \leq n$, then, $M_{1} \backslash\{i\} \neq M_{2} \backslash\{i\}$. In conclusion, there is $1-1$ correspondence between the terms on the left-hand side of (56) and the terms on the right-hand side. So (56) is valid.

Putting (56) in (55) we obtain

$$
\begin{aligned}
\sum_{\emptyset \neq M \subset\{1, \ldots, n\}} & \sum_{i \notin M} B_{i}^{2} S_{M \backslash\{i\}}\left(t-\tau_{i}\right) \\
- & \sum_{\emptyset \neq M \subset\{1, \ldots, n\}} \sum_{i \in M} B_{i}^{2} S_{M \backslash\{i\}}\left(t-\tau_{i}\right) \\
= & \sum_{M=\{1, \ldots, n\}} \sum_{i \notin M} B_{i}^{2} S_{M \backslash\{i\}}\left(t-\tau_{i}\right) \\
& -\sum_{M \in\{\{1\}, \ldots,\{n\}\}} \sum_{i \in M} B_{i}^{2} S_{M\{i\}}\left(t-\tau_{i}\right) .
\end{aligned}
$$

Next, by the property of empty sum, we get

$$
\sum_{M=\{1, \ldots, n\}} \sum_{i \notin M} B_{i}^{2} S_{M \backslash\{i\}}\left(t-\tau_{i}\right)=\sum_{M=\{1, \ldots, n\}} \Theta=\Theta .
$$

Moreover, it holds

$$
\begin{aligned}
& \sum_{M \in\{\{1\}, \ldots,\{n\}\}} \sum_{i \in M} B_{i}^{2} S_{M \backslash\{i\}}\left(t-\tau_{i}\right) \\
& =\sum_{i=1}^{n} B_{i}^{2} S_{\emptyset}\left(t-\tau_{i}\right)=\sum_{M=\emptyset} \sum_{i=1}^{n} B_{i}^{2} S_{M}\left(t-\tau_{i}\right) .
\end{aligned}
$$

Therefore, putting (60) and (61) in (59) and the result in (53), we obtain

$$
\begin{aligned}
\ddot{X}_{\tau_{1}, \ldots, \tau_{n}}^{B_{1}^{2}, \ldots, \tau_{n}^{2}}(t)= & -\sum_{\emptyset \neq M \subset\{1, \ldots, n\}} \sum_{i=1}^{n} B_{i}^{2} S_{M}\left(t-\tau_{i}\right) \\
& -\sum_{M=\emptyset} \sum_{i=1}^{n} B_{i}^{2} S_{M}\left(t-\tau_{i}\right) \\
= & -\sum_{i=1}^{n} B_{i}^{2} \sum_{M \subset\{1, \ldots, n\}} S_{M}\left(t-\tau_{i}\right) \\
= & -\sum_{i=1}^{n} B_{i}^{2} x_{\tau_{1}, \ldots, \tau_{n}}^{B_{1}^{2}, \ldots B_{n}^{2}}\left(t-\tau_{i}\right) .
\end{aligned}
$$

Hence, $\mathscr{X}_{\tau_{1}, \ldots, \tau_{n}}^{B_{1}^{2}, \ldots, B_{n}^{2}}(t)$ solves (31) for all $t \geq 0$. Clearly, the same is true for $t<0$.
For $\mathcal{Y}_{\tau_{1}, \ldots, \tau_{n}}^{B_{1}^{2}, \ldots, B_{n}^{2}}(t)$, statements (1)-(3) can be proved as for $\mathscr{X}_{\tau_{1}, \ldots, \tau_{n}}^{B_{1}^{2}, \ldots, B_{n}^{2}}(t)$. Next, if $\tau:=\tau_{1}=\cdots=\tau_{n}$, we apply the point (2) of this lemma and property (22) for $\mathcal{Y}_{\tau}^{B_{1}^{2}+\cdots+B_{n}^{2}}(t)$ to see that

$$
\begin{aligned}
\ddot{\mathscr{Y}}_{\tau_{1}, \ldots, \tau_{n}}^{B_{1}^{2}, \ldots, B_{n}^{2}}(t)= & \ddot{\mathscr{Y}}_{\tau}^{B_{1}^{2}+\cdots+B_{n}^{2}}(t) \\
= & -\left(B_{1}^{2}+\cdots+B_{n}^{2}\right) \mathscr{Y}_{\tau}^{B_{1}^{2}+\cdots+B_{n}^{2}}(t-\tau) \\
= & -B_{1}^{2} \mathscr{Y}_{\tau_{1}, \ldots, \tau_{n}}^{B_{1}^{2}, \ldots, B_{n}^{2}}\left(t-\tau_{1}\right) \\
& -\cdots-B_{n}^{2} \mathscr{Y}_{\tau_{1}, \ldots, \tau_{n}}^{B_{1}^{2}, \ldots, B_{n}^{2}}\left(t-\tau_{n}\right) .
\end{aligned}
$$

So, $\mathcal{Y}_{\tau_{1}, \ldots, \tau_{n}}^{B_{1}^{2}, \ldots, B_{n}^{2}}(t)$ is a solution of (31) when all delays are the same.

Again, the case $n=2$ with different delays was proved in [16]; thus, we assume that the statement is fulfilled for $n-$ $1, n \geq 3$ and that $\tau_{i} \neq \tau_{j}$ for each $i \neq j, i, j \in\{1, \ldots, n\}$. As before, if $t<\tau_{k}$ and $\tau_{k}:=\max _{i=1, \ldots, n} \tau_{i}$, then

$$
\mathcal{Y}_{\tau_{1}, \ldots, \tau_{n}}^{B_{1}^{2}, \ldots, B_{n}^{2}}(t)=\mathcal{Y}_{\tau_{1}, \ldots, \tau_{k-1}, \tau_{k+1}, \ldots, \tau_{n}}^{B_{1}^{2}, \ldots, B_{k-1}^{2}, B_{k}^{2}, \ldots, B_{n}^{2}}(t)
$$

by definition (20), and the statement follows from the inductive hypothesis. For $t \geq \max _{i=1, \ldots, n} \tau_{i}$, we apply Lemma 3 to see that

$$
\mathcal{Y}_{\tau_{1}, \ldots, \tau_{n}}^{B_{1}^{2}, \ldots, B_{n}^{2}}(t)=\sum_{M \subset\{1, \ldots, n\}} \widetilde{S}_{M}(t)
$$

with $\widetilde{S}_{M}(t)$ given by (25). This time

$$
\begin{aligned}
S_{\emptyset}(t) & =\sum_{\substack{j_{i} \geq 1, i \in \emptyset \\
0 \leq t}}(-1)^{0}\left(\left\{j_{i} \mid i \in \emptyset\right\}\right) ! E \frac{(t-0)^{1}}{0 !} \\
& =\sum_{0 \leq t} E t=t E \chi_{[0, \infty)}(t)
\end{aligned}
$$

and $S_{\emptyset}^{\prime \prime}(t)=\Theta$. The rest proceeds analogically to $\mathscr{X}_{\tau_{1}, \ldots, \tau_{n}}^{B_{1}^{2}, \ldots, B_{n}^{2}}(t)$.

The final statement follows directly from definition (20).

Remark 5. Another proof of statements (1)-(3) of the previous lemma can be made with the aid of statement (4) of the same lemma and uses the uniqueness of a solution of the corresponding initial value problem. For instance in statement (1) of the lemma, both

$$
\mathscr{X}_{\tau_{1}, \ldots, \tau_{n}}^{B_{1}^{2}, \ldots, B_{n}^{2}}(t), \quad X_{\tau_{1}, \ldots, \tau_{i-1}, \tau_{i+1}, \ldots, \tau_{n}}^{B_{1}^{2}, \ldots, B_{i-1}^{2}, B_{i+1}^{2}, \ldots, B_{n}^{2}}(t)
$$

solve

$$
\begin{aligned}
\ddot{x}(t)= & -B_{1}^{2} x\left(t-\tau_{1}\right)-\cdots-B_{i-1}^{2} x\left(t-\tau_{i-1}\right) \\
& -B_{i+1}^{2} x\left(t-\tau_{i+1}\right)-\cdots-B_{n}^{2} x\left(t-\tau_{n}\right)
\end{aligned}
$$

with initial condition

$$
x(t)=\left\{\begin{array}{ll}
\Theta, & -\tau \leq t<0, \\
E, & t=0,
\end{array} \dot{x}(t)=\Theta,-\tau \leq t \leq 0\right.
$$

and $\tau=\max _{i=1, \ldots, n} \tau_{i}$.

We are ready to state and prove our main result. 


\section{Main Result}

Here we find a solution of the initial value problem (11), (8) in the sense of the next definition.

Definition 6. Let $\tau_{1}, \ldots, \tau_{n}>0, \tau:=\max _{i=1, \ldots, n} \tau_{i}$, and $\varphi \in$ $C^{1}\left([-\tau, 0], \mathbb{R}^{N}\right)$, and let $B_{1}, \ldots, B_{n}$ be $N \times N$ matrices, and let $f:[0, \infty) \rightarrow \mathbb{R}^{N}$ be a given function. Function $x:$ $[-\tau, \infty) \rightarrow \mathbb{R}^{N}$ is a solution of (11) and initial condition (8), if $x \in C^{1}\left([-\tau, \infty), \mathbb{R}^{N}\right) \cap C^{2}\left([0, \infty), \mathbb{R}^{N}\right)$ (taken the second right-hand derivative at 0$)$ satisfies $(11)$ on $[0, \infty)$ and condition $(8)$ on $[-\tau, 0]$.

Theorem 7. Let $n \geq 3, \tau_{1}, \ldots, \tau_{n}>0, \tau:=\max _{i=1, \ldots, n} \tau_{i}$, and $\varphi \in C^{1}\left([-\tau, 0], \mathbb{R}^{N}\right)$, and let $B_{1}, \ldots, B_{n}$ be $N \times N$ pairwise permutable matrices; that is, $B_{i} B_{j}=B_{j} B_{i}$ for each $i, j \in$ $\{1, \ldots, n\}$, and let $f:[0, \infty) \rightarrow \mathbb{R}^{N}$ be a given function. Solution $x(t)$ of (11) satisfying initial condition (8) has the form

$$
x(t)= \begin{cases}\varphi(t), & -\tau \leq t<0, \\ \mathscr{X}(t) \varphi(0)+\mathscr{Y}(t) \dot{\varphi}(0) & \\ \quad-\sum_{i=1}^{n} B_{i}^{2} \int_{-\tau_{i}}^{0} \mathscr{Y}\left(t-\tau_{i}-s\right) \varphi(s) d s & \\ \quad+\int_{0}^{t} \mathscr{Y}(t-s) f(s) d s, & 0 \leq t,\end{cases}
$$

where $\mathscr{X}(t)=\mathscr{X}_{\tau_{1}, \ldots, \tau_{n}}^{B_{1}^{2}, \ldots, B_{n}^{2}}(t)$ and $\mathcal{Y}(t)=\mathscr{Y}_{\tau_{1}, \ldots, \tau_{n}}^{B_{1}^{2}, \ldots, B_{n}^{2}}(t)$.

Proof. Obviously, $x(t)$ satisfies the initial condition on $[-\tau, 0)$, and, from definition (20), $x(0)=\varphi(0)$. For the derivative, it holds $\lim _{t \rightarrow 0^{-}} \dot{x}(t)=\dot{\varphi}(0)$. Moreover, if $0 \leq t<\min _{i=1, \ldots, n} \tau_{i}$, then

$$
\begin{aligned}
x(t)= & \varphi(0)+t \dot{\varphi}(0) \\
& -\sum_{i=1}^{n} B_{i}^{2} \int_{-\tau_{i}}^{t-\tau_{i}}\left(t-\tau_{i}-s\right) \varphi(s) d s \\
& +\int_{0}^{t}(t-s) f(s) d s
\end{aligned}
$$

since

$$
\mathscr{Y}\left(t-\tau_{i}-s\right)= \begin{cases}\left(t-\tau_{i}-s\right) E, & s \in\left[-\tau_{i}, t-\tau_{i}\right], \\ \Theta, & s \in\left(t-\tau_{i}, 0\right]\end{cases}
$$

for each $i=1, \ldots, n$. Thus

$$
\dot{x}(t)=\dot{\varphi}(0)-\sum_{i=1}^{n} B_{i}^{2} \int_{-\tau_{i}}^{t-\tau_{i}} \varphi(s) d s+\int_{0}^{t} f(s) d s
$$

and $\lim _{t \rightarrow 0^{+}} \dot{x}(t)=\dot{\varphi}(0)$. Clearly,

$$
x \in C^{1}\left((-\tau, \infty), \mathbb{R}^{N}\right) \cap C^{2}\left((0, \infty) \backslash\left\{\tau_{1}, \ldots, \tau_{n}\right\}, \mathbb{R}^{N}\right) .
$$

We show that, although $\mathscr{X}(t)$ is not $C^{2}$ at $\tau_{1}, \ldots, \tau_{n}$, function $x(t)$ is $C^{2}$ at these points and, therefore, in $(0, \infty)$. At once, we prove that $x(t)$ is a solution of (11).
Assume that $0 \leq t<\min _{i=1, \ldots, n} \tau_{i}$. Then identities (71) and (73) are valid, and by differentiating (73) for such $t$ we get

$$
\ddot{x}(t)=-\sum_{i=1}^{n} B_{i}^{2} \varphi\left(t-\tau_{i}\right)+f(t)=-\sum_{i=1}^{n} B_{i}^{2} x\left(t-\tau_{i}\right)+f(t)
$$

since $x\left(t-\tau_{i}\right)=\varphi\left(t-\tau_{i}\right)$ for each $i=1, \ldots, n$.

Now, let $\emptyset \neq M_{1,2} \subset\{1, \ldots, n\}$ be such that $\tau_{i} \leq t<\tau_{j}$ for each $i \in M_{1}, j \in M_{2}$. Then

$$
\mathscr{Y}\left(t-\tau_{j}-s\right)= \begin{cases}\mathscr{Y}\left(t-\tau_{j}-s\right), & s \in\left[-\tau_{j}, t-\tau_{j}\right], \\ \Theta, & s \in\left(t-\tau_{j}, 0\right]\end{cases}
$$

whenever $j \in M_{2}$, and (70) becomes

$$
\begin{aligned}
x(t)= & \mathscr{X}(t) \varphi(0)+\mathscr{Y}(t) \dot{\varphi}(0) \\
& -\sum_{i \in M_{1}} B_{i}^{2} \int_{-\tau_{i}}^{0} \mathscr{Y}\left(t-\tau_{i}-s\right) \varphi(s) d s \\
& -\sum_{j \in M_{2}} B_{j}^{2} \int_{-\tau_{j}}^{t-\tau_{j}} \mathscr{Y}\left(t-\tau_{j}-s\right) \varphi(s) d s \\
& +\int_{0}^{t} \mathcal{Y}(t-s) f(s) d s .
\end{aligned}
$$

By the point (5) of Lemma 4, we get

$$
\begin{aligned}
\dot{x}(t)= & \dot{\mathscr{X}}(t) \varphi(0)+\dot{\mathscr{Y}}(t) \dot{\varphi}(0) \\
& -\sum_{i \in M_{1}} B_{i}^{2} \int_{-\tau_{i}}^{0} \dot{\mathscr{Y}}\left(t-\tau_{i}-s\right) \varphi(s) d s \\
& -\sum_{j \in M_{2}} B_{j}^{2} \int_{-\tau_{j}}^{t-\tau_{j}} \mathscr{X}\left(t-\tau_{j}-s\right) \varphi(s) d s \\
& +\int_{0}^{t} \mathscr{X}(t-s) f(s) d s,
\end{aligned}
$$

and for the second derivative it holds

$$
\begin{aligned}
\ddot{x}(t)= & \ddot{X}(t) \varphi(0)+\ddot{\mathscr{Y}}(t) \dot{\varphi}(0) \\
& -\sum_{i \in M_{1}} B_{i}^{2} \int_{-\tau_{i}}^{0} \ddot{\mathscr{Y}}\left(t-\tau_{i}-s\right) \varphi(s) d s \\
& -\sum_{j \in M_{2}} B_{j}^{2}\left(\varphi\left(t-\tau_{j}\right)+\int_{-\tau_{j}}^{t-\tau_{j}} \ddot{\mathscr{Y}}\left(t-\tau_{j}-s\right) \varphi(s) d s\right) \\
& +f(t)+\int_{0}^{t} \ddot{\mathscr{Y}}(t-s) f(s) d s
\end{aligned}
$$

since $\mathscr{X}(0)=E$. Now, we apply the property (4) of Lemma 4 together with

$$
\mathscr{X}\left(t-\tau_{j}\right)=\mathscr{Y}\left(t-\tau_{j}\right)=\Theta, \quad \forall j \in M_{2}
$$


to see that both $\mathscr{X}$ and $\mathscr{Y}$ are solutions of

$$
\ddot{y}(t)=-\sum_{i \in M_{1}} B_{i}^{2} y\left(t-\tau_{i}\right) .
$$

Therefore,

$$
\begin{aligned}
& \ddot{x}(t) \\
& =-\sum_{k \in M_{1}} B_{k}^{2}\left(\mathscr{X}\left(t-\tau_{k}\right) \varphi(0)+\mathscr{Y}\left(t-\tau_{k}\right) \dot{\varphi}(0)\right. \\
& -\sum_{i \in M_{1}} B_{i}^{2} \int_{-\tau_{i}}^{0} \mathscr{Y}\left(t-\tau_{i}-\tau_{k}-s\right) \varphi(s) d s \\
& -\sum_{j \in M_{2}} B_{j}^{2} \int_{-\tau_{j}}^{t-\tau_{j}} \mathscr{Y}\left(t-\tau_{j}-\tau_{k}-s\right) \varphi(s) d s \\
& \left.+\int_{0}^{t} \mathscr{Y}\left(t-\tau_{k}-s\right) f(s) d s\right) \\
& -\sum_{j \in M_{2}} B_{j}^{2} \varphi\left(t-\tau_{j}\right)+f(t) \\
& =-\sum_{i \in M_{1}} B_{i}^{2} x\left(t-\tau_{i}\right)-\sum_{j \in M_{2}} B_{j}^{2} \varphi\left(t-\tau_{j}\right)+f(t) .
\end{aligned}
$$

In fact, this is exactly formula (11) since $x\left(t-\tau_{j}\right)=\varphi\left(t-\tau_{j}\right)$ for each $j \in M_{2}$.

Finally, if $\max _{i=1, \ldots, n} \tau_{i} \leq t$, we have

$$
\begin{aligned}
x(t)= & \mathscr{X}(t) \varphi(0)+\mathscr{Y}(t) \dot{\varphi}(0) \\
& -\sum_{i=1}^{n} B_{i}^{2} \int_{-\tau_{i}}^{0} \mathscr{Y}\left(t-\tau_{i}-s\right) \varphi(s) d s \\
& +\int_{0}^{t} \mathscr{Y}(t-s) f(s) d s .
\end{aligned}
$$

So, differentiating this formula twice and applying (4) of Lemma 4 result in (11). Hence, one can see that function $x(t)$ given by (70) really solves (11) and satisfies initial condition (8) and, moreover, that $x \in C^{2}\left((0, \infty), \mathbb{R}^{N}\right)$. To see the last one, one has to put $\tau_{1}, \ldots, \tau_{n}$ into the computed derivatives, for example, if $\tau_{k}:=\min _{i=1, \ldots, n} \tau_{i}<\tau_{i}$ for each $i=1, \ldots, k-$ $1, k+1, \ldots, n$, then by (75) and (82) we get

$$
\begin{aligned}
\lim _{t \rightarrow \tau_{k}^{-}} \ddot{x}(t)= & -\sum_{\substack{i=1 \\
i \neq k}}^{n} B_{i}^{2} \varphi\left(\tau_{k}-\tau_{i}\right)-B_{k}^{2} \varphi(0)+f\left(\tau_{k}\right) \\
= & -\sum_{j \in M_{2}} B_{j}^{2} \varphi\left(\tau_{k}-\tau_{j}\right) \\
& -B_{k}^{2} x(0)+f\left(\tau_{k}\right)=\lim _{t \rightarrow \tau_{k}^{+}} \ddot{x}(t)
\end{aligned}
$$

where $M_{2}=\{1, \ldots, n\} \backslash\{k\}$.
It is easy to see that defining functions

$$
\begin{aligned}
& \widetilde{\mathscr{X}}_{\tau_{1}, \ldots, \tau_{n}}^{B_{1}, \ldots, B_{n}}(t):=\mathscr{X}_{\tau_{1}, \ldots, \tau_{n}}^{-B_{1}, \ldots,-B_{n}}(t), \\
& \widetilde{\mathscr{Y}}_{\tau_{1}, \ldots, \tau_{n}}^{B_{1}, \ldots, B_{n}}(t):=\mathscr{Y}_{\tau_{1}, \ldots, \tau_{n}}^{-B_{1}, \ldots, B_{n}}(t)
\end{aligned}
$$

leads to the solution of

$$
\ddot{x}(t)=B_{1} x\left(t-\tau_{1}\right)+\cdots+B_{n} x\left(t-\tau_{n}\right)+f(t)
$$

with pairwise permutable matrices $B_{1}, \ldots, B_{n}$ and initial condition (8). More precisely, we have the following corollary of Theorem 7 .

Corollary 8. Let $n \geq 3, \tau_{1}, \ldots, \tau_{n}>0, \tau:=\max _{i=1, \ldots, n} \tau_{i}$, $\varphi \in C^{1}\left([-\tau, 0], \mathbb{R}^{N}\right)$, and let $B_{1}, \ldots, B_{n}$ be $N \times N$ pairwise permutable matrices; that is, $B_{i} B_{j}=B_{j} B_{i}$ for each $i, j \in$ $\{1, \ldots, n\}$, and let $f:[0, \infty) \rightarrow \mathbb{R}^{N}$ be a given function. Solution $x(t)$ of (86) satisfying initial condition (8) has the form

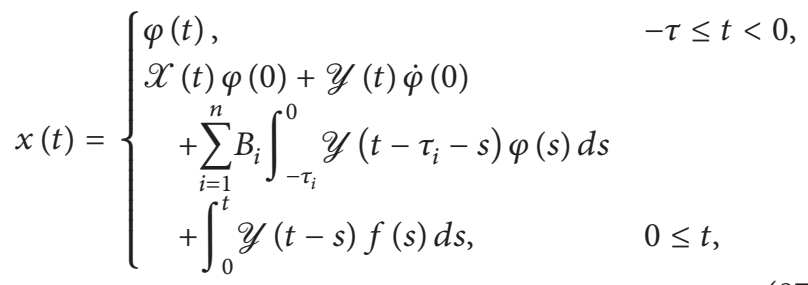

where $\mathscr{X}(t)=\widetilde{\mathscr{X}}_{\tau_{1}, \ldots, \tau_{n}}^{B_{1}, \ldots, B_{n}}(t)$ and $\mathscr{Y}(t)=\widetilde{\mathscr{Y}}_{\tau_{1}, \ldots, \tau_{n}}^{B_{1}, \ldots, B_{n}}(t)$.

Proof. The corollary can be proved exactly in the same way as Theorem 7.

\section{Acknowledgments}

J. Diblík was supported by the Grant GAČR P201/11/0768. M. Fečkan was supported in part by the Grants VEGAMS 1/0507/11, VEGA-SAV 2/0029/13, and APVV-0134-10. M. Pospíšil was supported by the Project no. CZ.1.07/2.3.00/ 30.0005 funded by European Regional Development Fund.

\section{References}

[1] D. Y. Khusainov, J. Diblík, M. Růžičková, and J. Lukáčová, "Representation of a solution of the Cauchy problem for an oscillating system with pure delay," Nonlinear Oscillations, vol. 11, no. 2, pp. 276-285, 2008.

[2] D. Y. Khusainov and G. V. Shuklin, "Linear autonomous timedelay system with permutation matrices solving," Studies of the University of Žilina, vol. 17, no. 1, pp. 101-108, 2003.

[3] M. Medved' and M. Pospíšil, "Sufficient conditions for the asymptotic stability of nonlinear multidelay differential equations with linear parts defined by pairwise permutable matrices," Nonlinear Analysis. Theory, Methods \& Applications, vol. 75, no. 7, pp. 3348-3363, 2012.

[4] M. Medved' and M. Pospíšil, "Representation and stability of solutions of systems of difference equations with multiple delays and linear parts defined by pairwise permutable matrices," 
Communications on Pure and Applied Analysis, vol. 17, pp. 2146, 2013.

[5] M. Pospíšil, "Representation and stability of solutions of systems of functional differential equations with multiple delays," Electronic Journal of Qualitative Theory of Differential Equations, no. 54, pp. 1-30, 2012.

[6] J. Diblík and D. Y. Khusainov, "Representation of solutions of linear discrete systems with constant coefficients and pure delay," Advances in Difference Equations, vol. 2006, Article ID 80825, 13 pages, 2006.

[7] A. Boichuk, J. Diblík, D. Khusainov, and M. Růžičková, "Boundary value problems for delay differential systems," Advances in Difference Equations, vol. 2010, Article ID 593834, 20 pages, 2010.

[8] A. Boichuk, J. Diblík, D. Khusainov, and M. Růžičková, "Fredholm's boundary-value problems for differential systems with a single delay," Nonlinear Analysis. Theory, Methods \& Applications, vol. 72, no. 5, pp. 2251-2258, 2010.

[9] A. Boichuk, J. Diblík, D. Khusainov, and M. Růžičková, "Boundary-value problems for weakly nonlinear delay differential systems," Abstract and Applied Analysis, vol. 2011, Article ID 631412, 19 pages, 2011.

[10] J. Diblík, D. Khusainov, O. Kukharenko, and Z. Svoboda, "Solution of the first boundary-value problem for a system of autonomous second-order linear partial differential equations of parabolic type with a single delay," Abstract and Applied Analysis, vol. 2012, Article ID 219040, 27 pages, 2012.

[11] J. Diblík, D. Y. Khusainov, J. Lukáčová, and M. Růžičková, "Control of oscillating systems with a single delay," Advances in Difference Equations, Article ID 108218, 15 pages, 2010.

[12] J. Diblík, D. Y. Khusainov, and M. Růžičková, "Controllability of linear discrete systems with constant coefficients and pure delay," SIAM Journal on Control and Optimization, vol. 47, no. 3, pp. 1140-1149, 2008.

[13] D. Y. Khusainov and G. V. Shuklin, "Relative controllability in systems with pure delay," International Applied Mechanics, vol. 41, no. 2, pp. 210-221, 2005.

[14] M. Medved', M. Pospíšil, and L. Škripková, "Stability and the nonexistence of blowing-up solutions of nonlinear delay systems with linear parts defined by permutable matrices," Nonlinear Analysis. Theory, Methods \& Applications, vol. 74, no. 12, pp. 3903-3911, 2011.

[15] M. Medved' and L. Škripková, "Sufficient conditions for the exponential stability of delay difference equations with linear parts defined by permutable matrices," Electronic Journal of Qualitative Theory of Differential Equations, no. 22, pp. 1-13, 2012.

[16] J. Diblík, M. Fečkan, and M. Pospíšil, "Representation of a solution of the Cauchy problem for an oscillating system with two delays and permutable matrices," Ukrainian Mathematical Journal, vol. 65, pp. 58-69, 2013.

[17] L. Chen and J. Sun, "Nonlinear boundary value problem of first order impulsive functional differential equations," Journal of Mathematical Analysis and Applications, vol. 318, no. 2, pp. 726-741, 2006.

[18] C. Li, J. Shi, and J. Sun, "Stability of impulsive stochastic differential delay systems and its application to impulsive stochastic neural networks," Nonlinear Analysis. Theory, Methods \& Applications, vol. 74, no. 10, pp. 3099-3111, 2011.

[19] M. Abramowitz and I. A. Stegun, Handbook of Mathematical Functions With Formulas, Graphs, and Mathematical Tables,
National Bureau of Standards, Washington, DC, USA, 10th edition, 1972. 


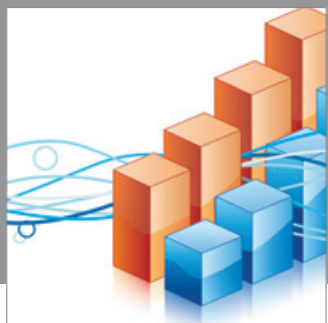

Advances in

Operations Research

mansans

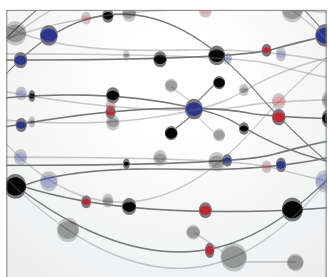

The Scientific World Journal
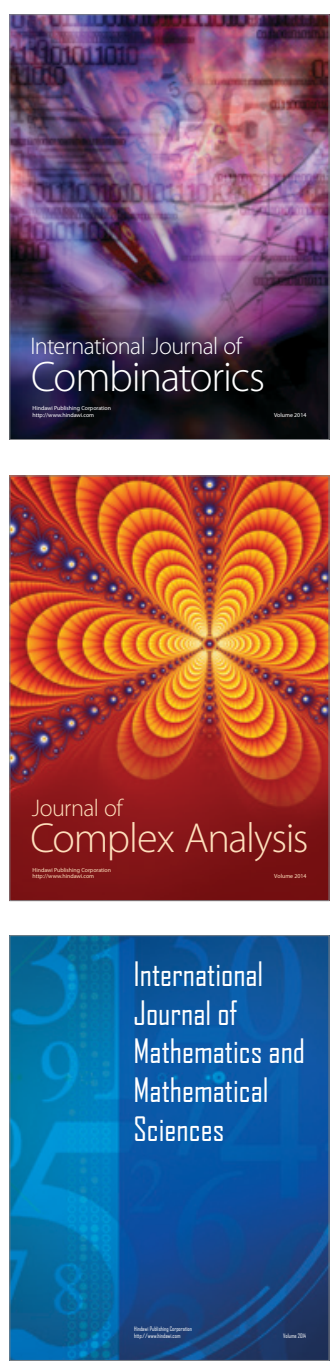
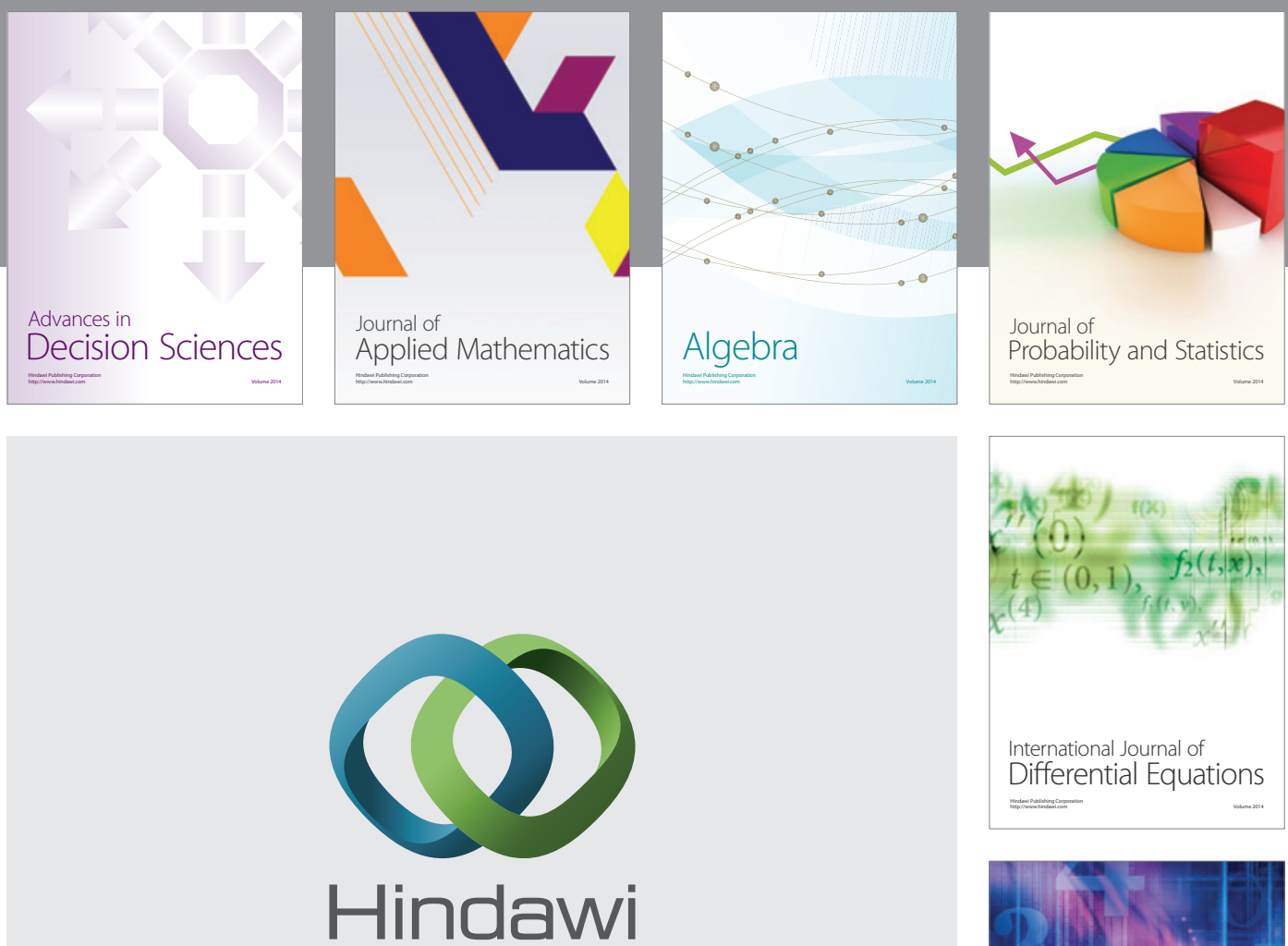

Submit your manuscripts at http://www.hindawi.com
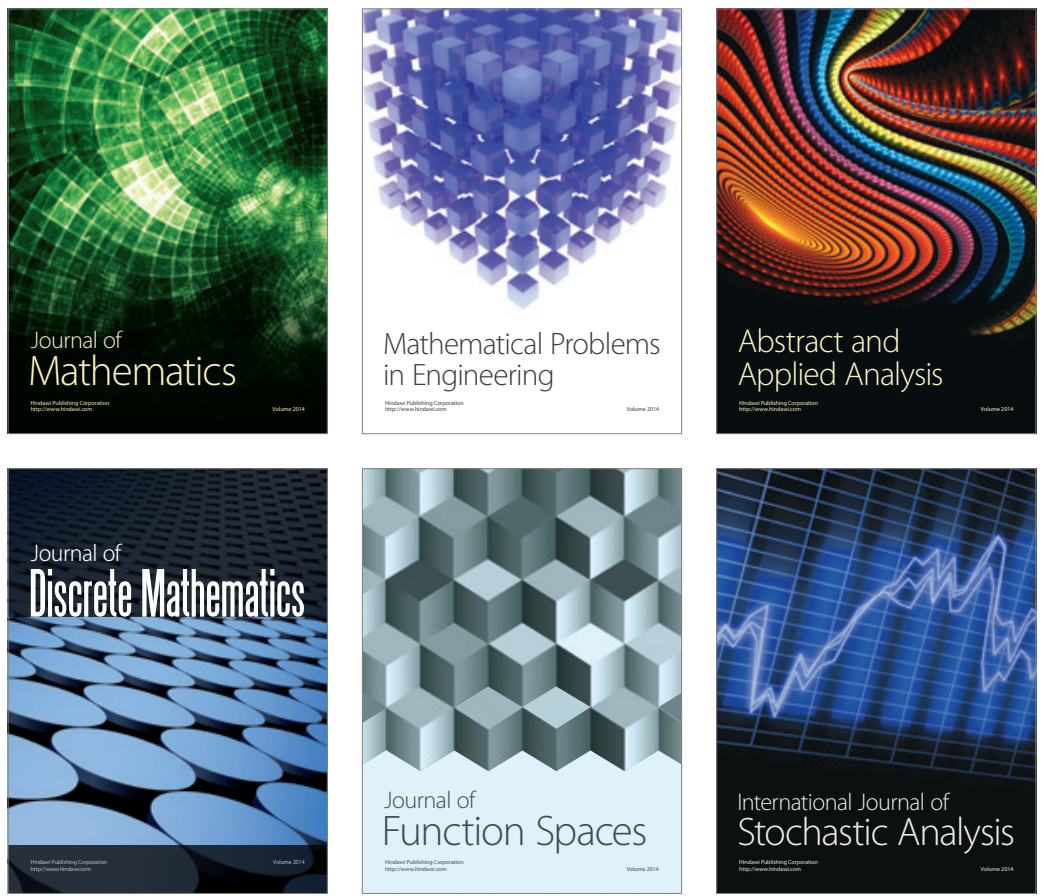

Journal of

Function Spaces

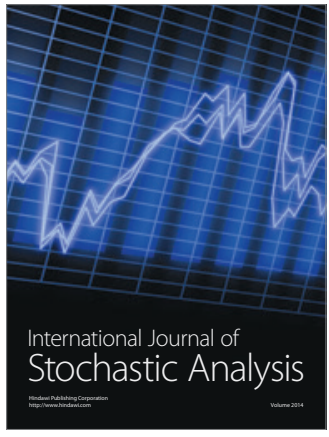

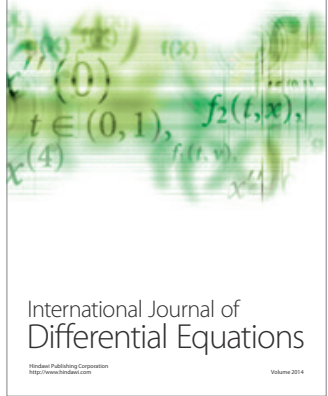
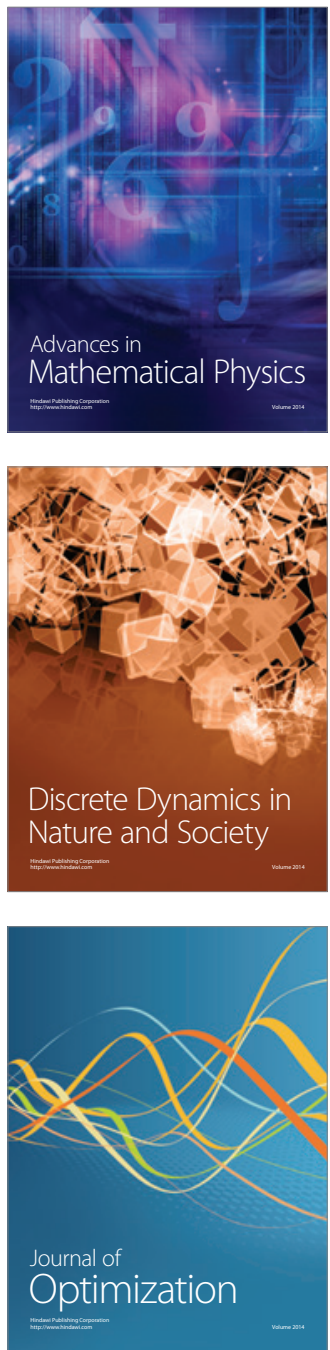